\title{
APPLICATION OF LAGUERRE POLYNOMIALS TO EVALUATION OF TWO-CENTER, TWO- AND THREE-ELECTRON INTEGRALS IN THE BASIS OF SLATER-TYPE ORBITALS - ALGORITHM AND NUMERICAL RESULTS
}

\section{J. BUDZIŃSKI}

Institute of Physics, University of Szczecin, Wielkopolska 15, 70-451 Szczecin, Poland M. FIRSZT AND W. WoŹNICKI

Institute of Physics, Nicolai Copernicus University

Grudziądzka 5, 87-100 Toruń, Poland

(Received January 11, 1994; revised version June 28, 1994)

Numerical verification of the algorithm for evaluation of the two-center, two- and three-electron integrals with the correlation factors of the type $r_{12}^{k}$, elaborated by us previously is presented. The influence of different parameters on the accuracy of the expressions for evaluating the integrals is discussed on the basis of the numerical results obtained.

PACS numbers: $31: 15 .+\mathrm{q}$

\section{Introduction}

The allowance for the correlation factors in the form of non-negative powers of the interelectron-distance function in a variation wave function leads to the expressions for the Hamiltonian matrix elements which take the form of a combination of the multi-electron integrals. In the case of the formulation presented in [1-3], at most the eight-center and four-electron integrals have to be taken into account.

The knowledge of algorithms for computing appropriate integrals is necessary to perform calculations in the frame of that model. A remarkable progress [4-10] has been achieved in this field by creating a series of interesting methods for evaluating two-electron integrals with the operator $r_{12}^{-1}$, involving multicenter integrals - up to four-center ones. At present, however, there are no methods for evaluation of the above-mentioned integrals with the operators involving correlation factors of the type $r_{i j}^{k}$ for arbitrary molecule. 
The problem discussed in this paper is limited to two-atom molecules, therefore in this case the problem is reduced to two-center integrals only. Evaluation of some two-center integrals with the correlation factors of the type $r_{i j}^{k}$ has been discussed by Rothstein [11], and applied (with some modifications) by Clary [12], where, at a particular stage of calculation, one has to introduce a numerical integration. The problem, but limited to the two-electron integrals, has been also discussed by Guseinov et al. [13].

Analytical formulas for evaluating the two-center, two- and three-electron integrals in prolate spherical coordinates $[14,15]$ as well as the two- and three-electron integrals with the one-electron operator [16] have been obtained. The numerical tests performed [16] give positive results justifying the usefulness of that method in practical applications.

Another method, defined in spherical coordiuates and utilizing the expansion of the function $r_{12}^{k}$ by means of the generalized Laguerre polynomials, has been also elaborated [17, 18]. The analytical expressions for evaluating the two-center, one-, two-, three- and four-electron integrals defined in [3] as well as the integrals with one-electron operator have been obtained as a result of that method.

In this paper, the numerical verification of the algorithm given in [18] was performed for the case of the two- and three-electron integrals of the type

$$
\begin{aligned}
& I_{2}=\left\langle\phi_{\alpha_{1}} \phi_{\alpha_{2}}\left|r_{12}^{u}\right| \phi_{\alpha_{1}^{\prime}} \phi_{\alpha_{2}^{\prime}}\right\rangle, \\
& I_{3}=\left\langle\phi_{\alpha_{1}} \phi_{\alpha_{2}} \phi_{\alpha_{3}}\left|r_{12}^{u} r_{23}^{u}\right| \phi_{\alpha_{1}^{\prime}} \phi_{\alpha_{2}^{\prime}} \phi_{\alpha_{3}^{\prime}}\right\rangle .
\end{aligned}
$$

In the above expressions $r_{i j}$ are the interelectron-distance functions, and $u$ and $v$ are integers taking the values $-1,0,1,2, \ldots$ The functions $\phi_{\alpha}$ are the Slater-type orbitals [19] expressed by the coordinates of the local system with the origin in the center (atom) $A$ or $B$. The quantum numbers and exponent defining the Slater orbital are represented by the index $\alpha=(n, l, m, \zeta)$.

\section{The algorithm for evaluating two-center, two- and three-electron integrals}

As it is shown in [18], simplification of calculations requires the Slater orbitals to be grouped into the pairs relating to the coordinates of the same electron $\phi_{\alpha}^{*} \phi_{\alpha^{\prime}}$, where $\alpha=(n, l, m, \zeta)$ and $\alpha^{\prime}=\left(n^{\prime}, l^{\prime}, m^{\prime}, \zeta^{\prime}\right)$. As a result of the use of the Perkins formula [20] for the function $r_{i j}^{u}$, where the radial part is expanded into the double series of the generalized Laguerre polynomials, the following expression for the two-electron integrals $I_{2}$ was obtained:

$$
I_{2}=(-1)^{M_{1}} \delta_{M_{1},-M_{2}} \sum_{s=\left|M_{1}\right|}^{\min \left(L_{1}^{u}, L_{1}, L_{2}\right)} \tilde{U}_{s}(1) \boldsymbol{S}_{u, s} \boldsymbol{U}_{s}(2),
$$

where $M_{i}=m_{i}^{\prime}-m_{i}$. The upper limit of the summation in (3) should be a minimum value among the three quantities: $L_{1}^{u}$ which takes the value $u / 2$ if $u$ is even, and infinity if $u$ is odd; $L_{i}(i=1,2)$ - in dependence whether the orbitals in an $i$-th pair belong to the center $A$ or $B$ : if both the orbitals belong to the center $A$ (the case $[A A])$ then $L_{i}=l_{i}+l_{i}^{\prime}$, and in other cases $([A B],[B A]$ and $[B B]) L_{i}=\infty$. 
Therefore, except the particular cases, the summation over the index $s$ in Eq. (3) runs up to infinity.

The quantity $\boldsymbol{S}_{u, s}$ in Eq. (3) is a matrix with the elements being the coeffcients of the above-mentioned expansion of the radial parts of the Perkins formula into the double series of the Laguerre polynomials. The values of those coefficients can be calculated by means of Eq. (31) in [18]. For a given $u$, the set of matrices with respect to the index $s$ should be determined. For even $u$, the range of these matrices is equal to $u$; for other cases - infinity, but in practice it is approximated by a finite value assuming the computing accuracy desired. Moreover, the quantity $\boldsymbol{U}_{s}(i)$ in Eq. (3) is a vector with the following components:

$$
U_{s \mu}^{\nu}(i)=N_{i} N_{i}^{\prime} \int_{0}^{\infty} F_{s}\left(\alpha_{i}, \alpha_{i}^{\prime} ; r, R\right) L_{\mu}^{\nu}(r) \mathrm{d} r
$$

where $F_{s}\left(\alpha_{i}, \alpha_{i}^{\prime} ; r, R\right)$ is a function of the form dependent on the distribution of the Slater orbitals in the $i$-th pair with respect to the centers $A$ and $B$ (detailed expressions are given by Eqs. (23)-(26) in [18]); $L_{\mu}^{\nu}(r)$ are the generalized Laguerre polynomials of the range $\mu$ [21] with respect to the variable $r$, where $\nu$ is a constant, $\nu>-1$, and it may be treated as the parameter of the expansion; $N_{i}$ and $N_{i}^{\prime}$ are the normalization factors for the radial part of the Slater orbitals in a given pair.

The numerical calculations based on Eq. (3) lead to the conclusion that the choice of the generalized Laguerre polynomials $L_{\mu}^{\nu}(a r)$ as the base functions, where $a$ is a positive parameter, instead of $L_{\mu}^{\nu}(r)$ improves the numerical computations. In this case, we get the following expansion for the function $r_{12}^{k}$ :

$$
r_{12}^{k}=4 \pi \sum_{s=0}^{L_{1}^{k}} \sum_{m=-s}^{s} Y_{s}^{m^{*}}\left(\hat{r}_{1}\right) Y_{s}^{m}\left(\hat{r}_{2}\right) \sum_{\mu_{1}, \mu_{2}=0}^{L_{3}^{k}} s_{\mu_{1} \mu_{2}}^{k s \nu}(a, b) L_{\mu_{1}}^{\nu}\left(a r_{1}\right) L_{\mu_{2}}^{\nu}\left(b r_{2}\right) \text {, }
$$

where $Y_{s}^{m}(\hat{r})$ are spherical harmonics [19]; $L_{1}^{k}$ and $L_{3}^{k}$ take infinite values for odd $k$, and values equal to $k / 2$ and $k$, respectively, for even $k$. Moreover,

$$
\begin{aligned}
& S_{\mu_{1} \mu_{2}}^{k s \nu}(a, b)=\frac{(a b)^{\nu+1} \mu_{1} ! \mu_{2} !}{\left(\mu_{1}+\nu\right) !\left(\mu_{2}+\nu\right) !} \sum_{p_{1}=0}^{\min \left(\mu_{1}, k+\nu+1\right)} b^{\mu_{1}-p_{1}}\left(\begin{array}{c}
k+\nu+1 \\
p_{1}
\end{array}\right) \\
& \quad \times \sum_{p_{2}=0}^{\min \left(\mu_{2}, k+\nu+1\right)} a^{\mu_{2}-p_{2}}\left(\begin{array}{c}
k+\nu+1 \\
p_{2}
\end{array}\right) \frac{(-1)^{p_{1}+\dot{p}_{2}}(q-1) !}{\left(\mu_{1}-p_{1}\right) !\left(\mu_{2}-p_{2}\right) !} \sum_{t=0}^{L_{2}^{k}} C_{k s t} \\
& \quad \times\left[B\left(s+2 t+\nu+\mu_{1}-p_{1} ; q ; a, b\right)+B\left(s+2 t+\nu+\mu_{2}-p_{2}, q ; b, a\right)\right] .
\end{aligned}
$$

In the above expression, $L_{2}^{k}$ takes the values $(k+1) / 2$ and $k / 2-s$ for odd and even $k$, respectively, $q=k+2 \nu+2+\mu_{1}-p_{1}+\mu_{2}-p_{2}$,

$$
C_{k s t}=\frac{1}{k+2}\left(\begin{array}{c}
k+2 \\
2 t+1
\end{array}\right) \prod_{j=0}^{\min (s-1,(k+1) / 2)} \frac{2 t-k-2 j}{2 t+1+2 s-2 j}
$$

and

$$
B(n, m ; a, b)=\int_{0}^{1} \frac{x^{n} \mathrm{~d} x}{(a+b x)^{m}}
$$


is the integral computable by means of the following formula:

$$
B(n, m ; a, b)=\frac{n !}{(m-1) !} \sum_{i=0}^{\infty} \frac{(m-1+i) ! b^{i}}{(n+1+i) !(a+b)^{m+i}} .
$$

Because of the slow convergence of Eq. (9) for $a<b$, the integrals $B(n, m ; a, b)$ can be evaluated by means of the following recurrences:

$$
\begin{aligned}
& B(n, m ; a, b)=\frac{1}{(m-1) a}\left[\frac{1}{(a+b)^{m-1}}+(m-n-2) B(n, m-1 ; a, b)\right], \\
& B(n, m ; a, b)=\frac{1}{(n+1) a}\left[\frac{1}{(a+b)^{m-1}}+b(m-n-2) B(n+1, m ; a, b)\right], \\
& B(n, m ; a, b)=a B(n, m+1 ; a, b)+b B(n+1, m+1 ; a, b),
\end{aligned}
$$

if the integrals of fixed initial values $m$ and $n$ are previously computed.

Taking into account the above expansion of the function $r_{12}^{k}$, we get the modified expression for the integral $I_{2}$ :

$$
I_{2}=\delta_{M_{1},-M_{2}}(-1)^{M_{1}} \sum_{s=\left|M_{1}\right|}^{\min \left(L_{1}^{u}, L_{1}, L_{2}\right)} \tilde{\boldsymbol{V}}_{s}(1, a) \boldsymbol{S}_{u, s}(a, b) \boldsymbol{V}_{s}(2, b),
$$

where $\boldsymbol{S}_{u, s}(a, b)$ is the matrix with elements given by Eq. $(6)$, and $\boldsymbol{V}_{\boldsymbol{s}}(i, a)$ is a vector with the following components:

$$
V_{s \mu}^{\nu}(i, a)=N_{i} N_{i}^{\prime} \int_{0}^{\infty} F_{s}\left(\alpha_{i}, \alpha_{i}^{\prime} ; r, R\right) L_{\mu}^{\nu}(a r) \mathrm{d} r
$$

In the case $[A A]$, the integration existing in Eq. (14) leads to the following results:

$$
\begin{aligned}
& V_{s \mu}^{\nu}(i, a)=N_{i} N_{i}^{\prime} \frac{q_{i} !}{d_{i}^{q_{i}+1}} \\
& \quad \times \sum_{j=0}^{\min \left(\mu, q_{i}-\nu\right)}(-1)^{j}\left(\begin{array}{c}
q_{i}-\nu \\
j
\end{array}\right)\left(\begin{array}{c}
q_{i}+\mu-\nu \\
\mu-j
\end{array}\right)\left[\frac{d_{i}-a}{d_{i}}\right]^{\mu-j},
\end{aligned}
$$

if $\nu \leq q_{i}$, and

$$
\begin{aligned}
& V_{s \mu}^{\nu}(i, a)=N_{i} N_{i}^{\prime} \frac{q_{i} !}{d_{i}^{q_{i}+1}} \\
& \quad \times \sum_{j=0}^{\mu}(-1)^{j}\left(\begin{array}{c}
\nu-q_{i}+j-1 \\
j
\end{array}\right)\left(\begin{array}{c}
q_{i}+\mu-j \\
q_{i}
\end{array}\right)\left[\frac{d_{i}-a}{d_{i}}\right]^{\mu-j},
\end{aligned}
$$

if $\nu>q_{i}$, where $q_{i}=n_{i}+n_{i}^{\prime}$ and $d_{i}=\zeta_{i}+\zeta_{i}^{\prime}$.

In the other cases $([A B],[B A]$ and $[B B])$, the integration domain should be divided into two ones: $0 \leq r \leq R$ and $R \leq r \leq \infty$, where $R$ is the distance between the centers $A$ and $B$. As it results from the explicit form of the function $F_{s}\left(\alpha_{i}, \alpha_{i}^{\prime} ; r, R\right)$, the component $V_{s \mu}^{\nu}(i, a)$ of the vector $\boldsymbol{V}_{s}(i, a)$ is a linear combination of the integrals of the following type in the domain $0 \leq r \leq R$ :

$$
A(n, \rho, R)=\int_{0}^{R} r^{n} \mathrm{e}^{-\rho r} \mathrm{~d} r
$$


where $\rho \geq 0$ and $n \geq 0$. In the interval $R \leq r \leq \infty$, it is a linear combination of the integrals of the type

$$
E(n, \omega, R)=\int_{R}^{\infty} r^{n} \mathrm{e}^{-\omega r} \mathrm{~d} r,
$$

where $\omega>0$, and $n$ is an integer.

In order to illustrate the above discussion, the explicit expressions for the case $[A B]$ are given below. Using Eq. (24) in [18], we get

$$
\begin{array}{r}
V_{s \mu}^{\nu}(i, a)=N_{i} N_{i}^{\prime}(2 s+1)^{1 / 2} \sum_{\lambda=|s-l|}^{s+l} C^{s}\left(\lambda, m^{\prime} ; l, m\right) \\
\times \sum_{p=0}^{n^{\prime}} I\left(n, \lambda, p, \mu, \nu ; \zeta, \zeta^{\prime}, R\right) g_{\lambda p}\left(n^{\prime}, l^{\prime}, m^{\prime} ; r, R\right),
\end{array}
$$

where $C^{s}\left(\lambda, m^{\prime} ; l, m\right)$ are the Condon-Shortley parameters $[19,22]$, and

$$
\begin{aligned}
& I\left(n, \lambda, p, \mu, \nu ; \zeta, \zeta^{\prime}, R\right) \\
& = \begin{cases}\sum_{f=0}^{\infty} c_{\lambda+p, f} \zeta^{\lambda+2 p+2 f} \\
\times \sum_{k=0}^{\mu} t_{\mu k}^{\nu}(a) A(n+1+k+\lambda+2 p+2 f, \zeta, R) & \text { for } r \leq R, \\
(-1)^{p} \sum_{f=0}^{\lambda+p} s_{\lambda+p, f} \zeta^{\prime-\lambda-1+p} & \text { for } r \geq R, \\
\times \sum_{k=0}^{\mu} t_{\mu k}^{\nu}(a) E\left(n+1+k-\lambda-1, \zeta+\zeta^{\prime}, R\right) & \\
c_{l j}=\frac{2^{l}(f+l) !}{(2 f+2 l+1) ! f !}, \quad s_{l f}=\frac{(2 l-f) !}{2^{l-f}(l-f) ! f !},\end{cases}
\end{aligned}
$$

and

$$
t_{\mu k}^{\nu}(a)=\frac{(-a)^{k}}{k !}\left(\begin{array}{c}
\mu+\nu \\
\mu-k
\end{array}\right)
$$

The functions $g_{\lambda p}\left(n^{\prime}, l^{\prime}, m^{\prime} ; r, R\right)$, existing in Eq. (19), are defined by Eqs. (16)-(21) in $[18]$. For the cases $[B A]$ and $[B B]$, we get similar expressions using Eq. (25) and (26) in [18], respectively.

Taking into account the expansion (5) of the interelectron distance, we get the following form for the integral $I_{3}$ : 


$$
\begin{aligned}
I_{3}= & \delta_{M_{1},-M_{2}-M_{3}}(-1)^{M_{1}} \\
& \times \sum_{s_{1}=\left|M_{1}\right|}^{\min \left(L_{1}^{u}, L_{1}\right)} \sum_{s_{3}=\left|M_{3}\right|}^{\min \left(L_{1}^{v}, L_{3}\right)} \sum_{s_{2}=\left|s_{1}-s_{3}\right|}^{\min \left(s_{1}+s_{3}, L_{2}\right)}\left(2 s_{2}+1\right)^{1 / 2} C^{s_{2}}\left(s_{1}, M_{1} ; s_{3},-M_{3}\right) \\
& \times \tilde{V}_{s_{1}}(1, a) S_{u_{1} s_{1}}(a, b) M_{s_{2}}(2, b) S_{v, s_{3}}(b, c) V_{s_{3}}(3, c)
\end{aligned}
$$

where - in comparison with the expression for $I_{2}$ - new quantities $M_{s}(i, a)$ exist. $M_{s}(i, a)$ are the matrices with the components defined by

$$
M_{s \mu_{1} \mu_{2}}^{\nu}(i, a)=N_{i} N_{i}^{\prime} \int_{0}^{\infty} F_{s}\left(\alpha_{i}, \alpha_{i}^{\prime} ; r, R\right) L_{\mu_{1}}^{\nu}(a r) L_{\mu_{2}}^{\nu}(a r) \mathrm{d} r .
$$

In the case $[A A]$, these components $\underset{\min \left(\mu_{1}, q_{i}\right.}{\text { for }} q_{i} \leqslant \nu$, can be calculated as follows:

$$
\begin{gathered}
M_{s \mu_{1} \mu_{2}}^{\nu}(i, a)=N_{i} N_{i}^{\prime} \frac{q_{i} !}{d_{i}^{q_{i}+1}} \sum_{j=0}^{\min \left(\mu_{1}, q_{i} \underline{q}_{\nu}\right)}(-1)^{j}\left(\begin{array}{c}
q_{i}-\nu \\
j
\end{array}\right)\left(\begin{array}{c}
q_{i}+\mu_{1}-j \\
q_{i}
\end{array}\right) \\
\times \sum_{l=0}^{\min \left(\mu_{2}, q_{i}-\nu\right)}(-1)^{l}\left(\begin{array}{c}
q_{i}-\nu \\
l
\end{array}\right) \stackrel{\sum_{k=0}^{\min \left(\mu_{1}-j, \mu_{2}-l\right)}\left(\begin{array}{c}
\mu_{1}-j \\
k
\end{array}\right)}{\times\left(\begin{array}{c}
q_{i}+\mu_{1}+\mu_{2}-j-l-k \\
\mu_{2}-l-k
\end{array}\right)\left[\frac{2 a-d_{i}}{d_{i}}\right]^{k}\left[\frac{d_{i}-a}{d_{i}}\right]^{\mu_{1}+\mu_{2}-j-l-2 k}} .
\end{gathered}
$$

In the case $[A B]$, these components, similarly as those of the vector $V_{s}(i, a)$, are linear combinations of the integrals of the types $A(n, \rho, R)$ and $E(n, \omega, R)$. Computations of the integrals given by Eq. (24) are based on expressions being similar to Eqs. (19) and (20) with the difference consisting in existence of two Laguerre polynomials in Eq. (24) - in comparison with Eq. (14) where only one polynomial exists.

In final expressions for the integrals $I_{2}$ given by Eq. (13) and $I_{3}$ given by Eq. (23), the summation over indices $s$ and $s_{1}, s_{3}$, respectively, runs, in general, up to infinity.

\section{Numerical results and conclusions}

Using Eqs. (13) and (23), a series of numerical calculations was performed. The results of those calculations are collected in Tabs. I-V. All the results were obtained under assumptions that all Slater orbitals are of the type $\phi_{\alpha}=1 s(\zeta)$ and the distance $R$ between the centers $A$ and $B$ is equal to 2 a.u. The following convention of the integral notation is introduced here:

$$
\begin{aligned}
& \left\langle\phi_{\alpha_{1}} \phi_{\alpha_{2}}\left|r_{12}^{u}\right| \phi_{\alpha_{1}^{\prime}} \phi_{\alpha_{2}^{\prime}}\right\rangle=\left(\phi_{\alpha_{1}} \phi_{\alpha_{1}^{\prime}}\left|\phi_{\alpha_{2}} \phi_{\alpha_{2}^{\prime}}\right| u\right) \\
& \left\langle\phi_{\alpha_{1}} \phi_{\alpha_{2}} \phi_{\alpha_{3}}\left|r_{12}^{u} r_{23}^{v}\right| \phi_{\alpha_{1}^{\prime}} \phi_{\alpha_{2}^{\prime}} \phi_{\alpha_{3}^{\prime}}\right\rangle=\left(\phi_{\alpha_{1}} \phi_{\alpha_{1}^{\prime}}\left|\phi_{\alpha_{2}} \phi_{\alpha_{2}^{\prime}}\right| \phi_{\alpha_{3}} \phi_{\alpha_{3}^{\prime}} \mid u, v\right) .
\end{aligned}
$$

This convention makes it legible to refer the Slater orbitals in a pair to the centers $A$ and/or $B$. 
TABLE I

The convergence test for the integrals $I_{2}$ and $I_{3}$ with respect to the values of summation limits $s$ in Eq. (13), and $s_{1}, s_{3}$ in Eq. (23), for fixed parameters: $\nu=0, \mu_{\max }=40$ and $a=b=2.0$.

\begin{tabular}{|c|c|c|c|c|}
\hline a) & $(A B|A B| u)$ & & $(A B|A A| A B \mid u, v)$ & $(A B|A B| A B \mid u, v)$ \\
\hline & $u=-1$ & & \multicolumn{2}{|c|}{$u=-1, \quad v=-1$} \\
\hline$s=0$ & 0.1597872183 & $s_{1}=s_{3}=0$ & 0.0991905375 & 0.0527307638 \\
\hline$s=1$ & 0.1804344442 & $s_{1}=s_{3}=1$ & 0.1012922237 & 0.0603246175 \\
\hline$s=2$ & 0.1834601259 & $s_{1}=s_{3}=2$ & 0.1014053984 & 0.0615301846 \\
\hline$\vdots$ & . & & $\vdots$ & $\vdots$ \\
\hline$s=10$ & 0.1841562597 & $s_{1}=s_{3}=10$ & 0.1014172123 & 0.0618254225 \\
\hline$s=1.1$ & 0.1841563819 & $s_{1}=s_{3}=11$ & 0.1014172132 & 0.0618254753 \\
\hline$s=12$ & 0.1841564457 & $s_{1}=s_{3}=12$ & 0.1014172133 & 0.0618255028 \\
\hline$s=13$ & 0.1841564905 & $s_{1}=s_{3}=13$ & 0.1014172134 & 0.0618255176 \\
\hline$s=14$ & 0.1841565003 & $s_{1}=s_{3}=14$ & 0.1014172134 & 0.0618255259 \\
\hline$s=15$ & 0.1841565120 & $s_{1}=s_{3}=15$ & 0.1014172135 & 0.0618255303 \\
\hline \multirow[t]{2}{*}{$\begin{array}{c}\text { Comparison } \\
\text { values }^{b)}\end{array}$} & 0.1841564571 & & 0.1014171959 & 0.0618254269 \\
\hline & $u=1$ & & \multicolumn{2}{|c|}{$u=1, \quad v=1$} \\
\hline$s=0$ & 0.9656207784 & $s_{1}=s_{3}=0$ & 2.2922041292 & 1.4773595778 \\
\hline$s=1$ & 0.8565092404 & $s_{1}=s_{3}=1$ & 2.3295279872 & 1.3275115772 \\
\hline$s=2$ & 0.8520000162 & $s_{1}=s_{3}=2$ & 2.3296972670 & 1.3217790593 \\
\hline$\vdots$ & $\vdots$ & & $\vdots$ & \\
\hline$s=10$ & 0.8515400477 & $s_{1}=s_{3}=10$ & 2.3297015417 & 1.3212239297 \\
\hline$s=11$ & 0.8515400406 & $s_{1}=s_{3}=11$ & 2.3297015417 & 1.3212239215 \\
\hline$s=12$ & 0.8515400375 & $s_{1}=s_{3}=12$ & 2.3297015417 & 1.3212239180 \\
\hline$s=13$ & 0.8515400369 & $s_{1}=s_{3}=13$ & 2.3297015417 & 1.3212239163 \\
\hline$s=14$ & 0.8515400354 & $s_{1}=s_{3}=14$ & 2.3297015417 & 1.3212239155 \\
\hline$s=15$ & 0.8515400351 & $s_{1}=s_{3}=15$ & 2.3297015417 & 1.3212239150 \\
\hline Comparison & & & & \\
\hline values $^{b)}$ & 0.8515400349 & & 2.3297015417 & 1.3212239146 \\
\hline
\end{tabular}

a) All the Slater orbitals are of the type $1 s$ with $\zeta=1.0$.

b) The integral values obtained on the basis of the algorithm defined in $[15,16]$.

In general, the expressions for evaluating the integrals $I_{2}$ and $I_{3}$ depend on some parameters regarding the expansion of the function $r_{12}^{k}$. They are the summation limits of $s, \mu_{1}$ and $\mu_{2}$ in Eq. (5), running to infinity for odd $k$, the order $\nu$ of the Laguerre polynomials, as well as the expansion constants $a$ and $b$. In practice, the upper limit for values $\mu_{1}$ and $\mu_{2}$ is fixed as a value $\mu_{\max }$ determining the range of the matrix $S_{k, s}(a, b)$ with elements given by Eq. (6). 
TABLE II

The values of the two-center integrals $I_{2}$ for different values of the expansion parameter $\nu$, and fixed parameters: $\mu_{\max }=40, s_{\max }=15$, and $a=b=2.0$.

\begin{tabular}{|c|c|c|c|}
\hline a) & $(A A|A B| u)$ & $(A A|B B| u)$ & $(A B|A B| u)$ \\
\hline & \multicolumn{3}{|c|}{$u=-1$} \\
\hline$\nu=0$ & 0.3080364658 & 0.425974292 & 0.184156 \\
\hline$\nu=1$ & 0.308036465 & 0.425974292 & 0.184156 \\
\hline$\nu=2$ & 0.308036465 & 0.42597429 & 0.18415 \\
\hline$\nu=3$ & 0.308036 & 0.4259 & 0.18415 \\
\hline$\nu=5$ & 0.308 & 0.425 & 0.184 \\
\hline \multirow{3}{*}{$\begin{array}{c}\text { Comparison } \\
\text { values }^{b)}\end{array}$} & & & \\
\hline & 0.3080364658 & 0.4259742928 & 0.1841564571 \\
\hline & \multicolumn{3}{|c|}{$u=1$} \\
\hline$\nu=0$ & 1.4776890110 & 2.919487504 & 0.85154003 \\
\hline$\nu=1$ & 1.477689011 & 2.919487504 & 0.8515400 \\
\hline$\nu=2$ & 1.477689011 & 2.91948750 & 0.8515400 \\
\hline$\nu=3$ & 1.477689 & 2.91948 & 0.85154 \\
\hline$\nu=5$ & 1.4776 & 2.9194 & 0.8515 \\
\hline \multirow{2}{*}{$\begin{array}{c}\text { Comparison } \\
\left.\text { values }^{b}\right)\end{array}$} & & & \\
\hline & 1.4776890110 & 2.9194875040 & 0.8515400349 \\
\hline
\end{tabular}

$a), b)$ See footnotes at Tab. I.

Assuming some fixed values for the parameters other than $s, s_{1}$ and $s_{3}$, we get numerical results for the integrals $I_{2}$ and $I_{3}$, as it is shown in Tab. $I$ with respect to increasing values of the summation index $s$ in Eq. (13), and $s_{1}, s_{3}$ in Eq. (23). The so-called comparison values are the computation results obtained by means of the method presented in $[15,16]$, based on the Neumann expansion. In our opinion, they are rather accurate. As it is seen, the convergence is rather slow and the obtained results have only 5-7 exact significant digits for some integrals.

To investigate the influence of the parameter $\nu$ on the convergence of the expression (13), the computation for its different values were performed. The results are presented in Tab. II. As it can be seen, the best results are obtained for $\nu=0$; the increase in that parameter over the value 2 leads to essential worsening of the convergence of Eq. (13). 
TABLE III

The influence of parameters $a$ and $b$ on the computing accuracy for the integrals $I_{2}$ with fixed parameters: $\gamma_{1}=1.3, \gamma_{2}=0.8$, $\nu=0, \mu_{\max }=40$ and $s_{\max }=15$.

\begin{tabular}{|c|c|c|c|}
\hline a) & $(A A|A B| u)$ & $(A A|B B| u)$ & $\overline{(A B|A B| u)}$ \\
\hline$u=-1$ & & & \\
\hline$a=0.2, b=0.1$ & 0.26 & 0.27 & 0.205 \\
\hline$a=1.0, b=0.6$ & 0.2616447405 & 0.271926791 & 0.205031 \\
\hline$a=1.3, b=0.8$ & 0.2616447405 & 0.2719267917 & 0.2050318 \\
\hline$a=1.5, b=1.0$ & 0.2616447405 & 0.2719267917 & 0.205031 \\
\hline$a=2.2, b=1.5$ & 0.261 & 0.271 & 0.205 \\
\hline$a=3.0, b=2.0$ & \multicolumn{3}{|c|}{ not convergent } \\
\hline Comparison values $\left.{ }^{b}\right)$ & 0.2616447405 & 0.2719267917 & 0.2050318 \\
\hline$u=1$ & & & \\
\hline$a=0.2, b=0.1$ & 4.2575 & 4.9099 & 3.311 \\
\hline$a=1.0, b=0.6$ & 4.2575101498 & 4.9099900977 & 3.3115325 \\
\hline$a=1.3, b=0.8$ & 4.2575101498 & 4.9099900977 & 3.3115325 \\
\hline$a=1.5, b=1.0$ & 4.2575101498 & 4.9099900977 & 3.3115325 \\
\hline$a=2.2, b=1.5$ & 4.2575101 & 4.90999009 & 3.311532 \\
\hline$a=3.0, b=2.0$ & \multicolumn{3}{|c|}{ not convergent } \\
\hline Comparison values $\left.{ }^{b}\right)$ & 4.2575101498 & 4.9099900977 & 3.31153255 \\
\hline
\end{tabular}

a) All the Slater orbitals are of the type $1 s$ with $\zeta_{1}=\zeta_{1}^{\prime}=0.65$ and $\zeta_{2}=\zeta_{2}^{\prime}=0.4$.

b) See footnote at Tab. I.

There is a strong influence of the parameters $a$ and $b$ on the convergence of Eq. (13) with regard to values of the Slater-orbital exponents $\zeta$ and the distance $R$. A convenient measure of the influence is the parameter $\gamma$ defined as follows:

$$
\gamma_{i}=R\left(\zeta_{i}+\zeta_{i}^{\prime}\right) / 2
$$

where $\zeta_{i}$ and $\zeta_{i}^{\prime}$ are the Slater-orbital exponents in the $i$-th pair. The computation results for the integrals $I_{2}$ for the fixed value $\gamma_{1}=1.3, \gamma_{2}=0.8$ and variable values of $a$ and $b$, are presented in Tab. III. As it is seen, the computing accuracy increases according to the decrease in the difference between values of the parameters $\gamma_{1}$ and $a ; \gamma_{2}$ and $b$. The optimum case takes place for $\gamma_{1} \approx a$ and $\gamma_{2} \approx b$, but expression (13) is divergent for $a \gg \gamma_{1}$ or/and $b \gg \gamma_{2}$. Similar conclusions concern Eq. (23) for the integrals $I_{3}$. 
TABLE IV

The values of the two-center integrals $I_{2}$ for different values of $\mu_{\max }$, and fixed parameters $\nu=0, \gamma_{1}=\gamma_{2}=2.0$, $s_{\max }=15$ and $a=b=2.0$.

\begin{tabular}{|c|c|c|c|}
\hline a) & $(A A|A B| u)$ & $(A A|B B| u)$ & $(A B|A B| u)$ \\
\hline & \multicolumn{3}{|c|}{$u=-1$} \\
\hline$\mu_{\max }=10$ & 0.308036 & 0.4259 & 0.184 \\
\hline$\mu_{\max }=20$ & 0.30803646 & 0.425974 & 0.1841 \\
\hline$\mu_{\max }=30$ & 0.3080364658 & 0.425974292 & 0.184156 \\
\hline$\mu_{\max }=40$ & 0.3080364658 & 0.4259742928 & 0.184156 \\
\hline Comparison & & & \\
\hline \multirow[t]{2}{*}{ values $^{b)}$} & 0.3080364658 & 0.4259742928 & 0.1841564571 \\
\hline & \multicolumn{3}{|c|}{$u=1$} \\
\hline$\mu_{\max }=10$ & 1.4776890 & 2.91948 & 0.8515 \\
\hline$\mu_{\max }=20$ & 1.477689011 & 2.91948750 & 0.85154 \\
\hline$\mu_{\max }=30$ & 1.4776890110 & 2.9194875040 & 0.85154003 \\
\hline$\mu_{\max }=40$ & 1.4776890110 & 2.9194875040 & 0.851540034 \\
\hline Comparison & & & \\
\hline values $\left.^{b}\right)$ & 1.4776890110 & 2.9194875040 & 0.8515400349 \\
\hline
\end{tabular}

$a), b)$ See footnotes at Tab. I.

The influence of the range $\mu$ of the matrices $S_{k, s}(a, b)$ on the integral values is illustrated in Tabs. IV and V for the integrals $I_{2}$ and $I_{3}$, respectively. In general, there is a strong influence of the parameter $\mu_{\max }$ on the computation accuracy which increases as the $\mu_{\max }$-value increases.

Numerical algorithm of computing two-center two- and three-electron integrals, defined in [18] and modified in this paper by taking into account the correction resulting from introducing the scaling factors $a$ and $b$ in the expansion of the radial part of the Perkins expression for the interelectron distance function $r_{12}^{k}$ into double series of Laguerre polynomials, was tested. This algorithm is a new proposition in relation to that defined in $[15,16]$ and based on the Neumann expansion for $r_{12}^{k}$. The comparison of the numerical results obtained in this work with those obtained previously $[15,16]$ allows to verify numerical values of the integrals discussed. The comparison of the results obtained by both methods confirms the correctness of the algorithm verified in the present paper. 
TABLE V

The values of the two-center integrals $I_{3}$ for different values of $\mu_{\max }$ and fixed parameters: $\nu=0, \gamma_{1}=\gamma_{2}=\gamma_{3}=2.0, s_{\max }=15$ and $a=b=c=2.0$.

\begin{tabular}{|c|c|c|c|c|}
\hline a) & $(A A|A A| A A \mid u, v)$ & $(A A|A A| A B \mid u, v)$ & $(A A|A B| A B \mid u, v)$ & $(A B|A B| A B \mid u, v)$ \\
\hline & \multicolumn{4}{|c|}{$u=-1, v=-1$} \\
\hline$\mu_{\max }=10$ & $0.4 \overline{259}$ & 0.2046 & 0.10 & 0.06 \\
\hline$\mu_{\max }=20$ & 0.4259259259 & 0.204649 & 0.10303 & 0.061 \\
\hline$\mu_{\max }=30$ & 0.4259259259 & 0.204649682 & 0.103030 & 0.0618 \\
\hline$\mu_{\max }=40$ & 0.4259259259 & 0.2046496820 & 0.1030305 & 0.061825 \\
\hline \multirow{3}{*}{$\begin{array}{c}\text { Comparison } \\
\left.\text { values }^{b}\right)\end{array}$} & & & & \\
\hline & 0.4259259259 & 0.2046496820 & 0.1030305895 & 0.0618254269 \\
\hline & \multicolumn{4}{|c|}{$u=-1, v=1$} \\
\hline$\mu_{\max }=10$ & 1.25810 & 0.868534 & 0.41994 & 0.250 \\
\hline$\mu_{\max }=20$ & 1.2581018518 & 0.868534469 & 0.4199423 & 0.2502 \\
\hline$\mu_{\max }=30$ & 1.2581018518 & 0.8685344697 & 0.41994235 & 0.250209 \\
\hline$\mu_{\max }=40$ & 1.2581018518 & 0.8685344697 & 0.419942357 & 0.2502097 \\
\hline \multirow{3}{*}{$\begin{array}{c}\text { Comparison } \\
\left.\text { values }^{b}\right)\end{array}$} & & & & \\
\hline & 1.2581018518 & 0.8685344697 & 0.4199423573 & 0.2502097521 \\
\hline & \multicolumn{4}{|c|}{$u=1, v=1$} \\
\hline$\mu_{\max }=10$ & 5.193287 & 3.442778 & 2.28740 & 1.32122 \\
\hline$\mu_{\max }=20$ & 5.1932870370 & 3.4427785 & 2.287406 & 1.321223 \\
\hline$\mu_{\max }=30$ & 5.1932870370 & 3.4427785891 & 2.28740687 & 1. 3212239 \\
\hline$\mu_{\max }=40$ & 5.1932870370 & 3.4427785891 & 2.287406876 & 1.321223914 \\
\hline \multirow{2}{*}{$\begin{array}{c}\text { Comparison } \\
\text { values } b)\end{array}$} & & & & \\
\hline & 5.1932870370 & 3.4427785891 & 2.2874068760 & 1.3212239146 \\
\hline
\end{tabular}

a), b) See footnotes at Tab. I.

\section{References}

[1] W. Woźnicki, in: Theory of Electronics Shells in Atoms and Molecules, Ed. A. Jucys, Mintis, Vilnius 1971, p. 103.

[2] J. Muszyńska, W. Woźnicki, Acta Phys. Pol. A 47, 113 (1975).

[3] A. Preiskorn, W. Woźnicki, Mol. Phys. 52, 1291 (1984).

[4] H. Tai, Phys. Rev. A 40, 6681 (1989).

[5] M.P. Barnett, Chem. Phys. Lett. 166, 65 (1990).

[6] E.N. Maslen, M.G. Trefry, Int. J. Quantum Chem. 37, 51 (1990).

[7] J. Fermandez Rico, R. Lopez, G. Ramirez, J. Chem. Phys. 94, 5032 (1991).

[8] H.W. Jones, Int. J. Quantum Chem. 42, 779 (1992).

[9] R. Montagnani, O. Salvetti, Int. J. Quantum Chem. 43, 273 (1992).

[10] V.I. Perevozchikov, I.V. Maslov, A.W. Niukkanen, H.H.H. Homeier, E.O. Steinborn, Int. J. Quantum Chem. 44, 45 (1992).

[11] E.V. Rothstein, Phys. Rev. A 3, 1581 (1971).

[12] D.C. Clary, Mol. Phys. 34, 793 (1977). 
[13] I.I. Guseinov, T.M. Mursalov, F.M. Dzhafarov, E.M. Imamov, Zh. Strukt. Khim. 20, 1129 (1979).

[14] J. Budzinski, Int. J. Quantum Chem. 28, 853 (1985).

[15] J. Budziński, Int. J. Quantum Chem. 41, 339 (1992).

[16] J. Budziński, M. Firszt, W. Woźnicki, Int. J. Quantum Chem. 41, 359 (1992).

[17] J. Budziński, Zesz. Nauk. Uniwersytetu Szczecińskiego 32, 28 (1988) (in English).

[18] J. Budziński, M. Firszt, W. Woźnicki, Acta Phys. Pol. A 78, 841 (1990).

[19] E.U. Condon, G.H. Shortley, The Theory of Atomic Spectra, University Press, Cambridge 1957.

[20] J.F. Perkins, J. Chem. Phys. 48, 1985 (1968).

[21] N.N. Lebiediew, Funkcje specjalne $i$ ich zastosowania, PWN, Warszawa 1957 (in Polish).

[22] D.A. Warszalowicz, A.N. Moskalev, W.K. Chersonski, Kvantovaya teorya uglogo momenta, Nauka, Leningrad 1975 (in Russian). 